\title{
Wiederholung und Wahrheit
}

\author{
Wie die mehrmalige Präsentation politischer Slogans die Einschätzung ihrer \\ Glaubwürdigkeit beeinflusst
}

\section{Thomas Koch / Thomas Zerback}

Der aus der Wabrnehmungspsychologie bekannte Truth-Effekt besagt, dass Personen Aussagen eine höbere Glaubwürdigkeit beimessen, wenn sie diese wiederholt wabrnehmen. Die vorliegende Studie untersucht diesen Zusammenhang erstmals im Kontext politischer Kommunikation. Im Rabmen eines experimentellen Designs präsentieren wir Probanden verschiedene Wablkampfslogans, wobei wir die Häufigkeit des Auftretens eines der Slogans gezielt manipulieren. Die Daten belegen den vermuteten Anstieg der Glaubwürdigkeit, allerdings nur bei Personen, die über themenspezifisches Vorwissen verfügen. Zudem deutet sich ein umgekehrt U-förmiger Zusammenhang zwischen Präsentationshäufigkeit und wabrgenommener Glaubwürdigkeit an, d. h. Letztere nimmt zunächst zu, geht bei zu häufiger Präsentation der Aussage jedoch wieder zurück.

Schlagwörter: Truth-Effekt, politische Kommunikation, politische Werbung, Wahlplakate, Wiederholungswirkungen, Online-Experiment

\section{Einleitung}

„And if all others accepted the lie which the Party imposed - if all records told the same tale then the lie passed into history and became truth" (Orwell 1987: 88).

In George Orwells Roman „1984“ ist eine der zentralen Aufgaben des „Ministry of Truth“, eine systemkonforme Realität für die Bürger des fiktiven Staates Ozeanien zu konstruieren. Entsprechend instrumentalisiert das Regime die Massenmedien, die fortwährend die immer gleichen Botschaften verbreiten und die Menschen so von der Legitimation des Überwachungsstaats überzeugen. Die Vorstellung, dass Menschen bestimmte Aussagen für wahr halten, wenn man sie nur oft genug damit konfrontiert, ist so faszinierend wie beunruhigend und längst nicht nur ein literarisches Gedankenspiel. Tatsächlich existiert ein wahrnehmungspsychologischer Effekt, der genau diesen $\mathrm{Zu}$ sammenhang beschreibt, der „Truth-Effekt“: Rezipienten glauben eine Aussage eher, wenn man sie ihnen wiederholt präsentiert (Hasher, Goldstein \& Toppino 1977). Besondere Relevanz erlangt diese Tatsache im Kontext persuasiver Kommunikation, weil Aussagen, die Rezipienten für glaubwürdig halten, weitaus größere Wirkungen auf Einstellungen (und Verhalten) entfalten als Botschaften, deren Wahrheitsgehalt zweifelhaft ist. Vor diesem Hintergrund überrascht es, dass sich die Kommunikationswissenschaft bisher nicht mit dem Truth-Effekt beschäftigt hat - zumal sowohl Wiederholungswirkungen als auch Effekte der Glaubwürdigkeit schon häufig Gegenstand kommunikationswissenschaftlicher Forschung waren. Die Frage, inwiefern Wiederholung und Glaubwürdigkeit zusammenhängen, ignorierte man jedoch weitgehend.

Die vorliegende Studie widmet sich dieser Forschungslücke. Dabei konzentrieren wir uns auf den Bereich der politischen Kommunikation, wo der Glaubwürdigkeit spezifischer Aussagen unmittelbar demokratietheoretische Bedeutung zukommt. Nicht die realen Umstände bilden nämlich die Basis menschlichen Handelns, sondern deren subjektive Wahrnehmung; die individuelle Situationsdefinition entscheidet demnach über das Handeln und entsprechende Konsequenzen (Thomas \& Thomas 1928). Dieses sog. 
Thomas-Theorem gilt auch für politisches Handeln und damit z. B. für die Bewertung politischer Akteure und letztlich für die Wahlentscheidung. Entsprechend haben politische Akteure großes Interesse daran, ihre jeweiligen Standpunkte richtig oder wahr erscheinen zu lassen. Hinzu kommt, dass die wiederholte Darbietung bestimmter Aussagen Alltag in der politischen Kommunikation ist: Über verschiedene Kommunikationskanäle werden Rezipienten stetig mit den gleichen Botschaften konfrontiert. In Nachrichtensendungen, Talkshowauftritten, Werbespots, Plakat- und Anzeigenwerbung, bei Wahlkampfveranstaltungen etc. wiederholen politische Akteure bestimmte Aussagen fortwährend. Bewirkt diese mehrfache Darbietung, dass Rezipienten die entsprechenden Botschaften eher glauben? Ausgehend von der bestehenden psychologischen Forschung zum Truth-Effekt konzipieren wir ein Experiment, in welchem wir die Bewertung der Glaubwürdigkeit eines politischen Slogans in Abhängigkeit von dessen Präsentationshäufigkeit prüfen. Drei Fragen stehen dabei im Mittelpunkt:

- „Steigt die Glaubwürdigkeit eines politischen Slogans, wenn man ihn häufiger präsentiert?"

- „Inwiefern moderiert das themenspezifische Vorwissen der Teilnehmer den TruthEffekt?"

- „Ist der Truth-Effekt beliebig zu steigern oder ergeben sich Grenzen hinsichtlich des Zugewinns an Glaubwürdigkeit?"“

\section{Der Truth-Effekt}

\subsection{Beschreibung des Effekts}

„Der höchste Gipfel im nordafrikanischen Atlasgebirge ist der Toubkal im Süden Marokkos“. Wenn Personen den Wahrheitsgehalt dieses Statements einschätzen sollen, prüfen sie zunächst, ob die Aussage mit ihrem eigenen Wissen übereinstimmt oder diesem widerspricht. Doch nur wenige Nordafrikaspezialisten oder passionierte Kletterer können mit Gewissheit sagen, ob der höchste Gipfel im Atlasgebirge tatsächlich der Toubkal ist. ${ }^{1}$ Wer nicht über das relevante Wissen verfügt, zieht spezifische Kontextmerkmale heran, um den Wahrheitsgehalt eines Statements einzuschätzen. Rezipienten könnten z. B. die Glaubwürdigkeit der Quelle als Referenz nutzen: Ist der Kommunikator vielleicht ein ausgewiesener Experte auf dem Gebiet? Auch der jeweilige Rahmen, in dem die Aussage getätigt wird, beeinflusst die Einschätzung des Wahrheitsgehalts: Erfolgt die Äußerung z. B. auf einer Fachkonferenz oder am Stammtisch? Zudem wirken sich Merkmale der Botschaft selbst auf deren Glaubwürdigkeitseinschätzung aus, beispielsweise eine schlüssige Argumentation. Indes bedarf es nicht unbedingt einer vertrauenswürdigen Quelle oder logischer Argumente, damit eine Aussage glaubwürdiger wirkt - unter Umständen reicht allein deren wiederholte Darbietung. Ein solcher Mechanismus klingt zunächst irrational, und man fühlt sich an die Metapher von Ludwig Wittgenstein erinnert, in der ein Mann mehrere Ausgaben der gleichen Zeitung kauft, um den Wahrheitsgehalt des ersten Exemplars zu überprüfen (Ayer 1986: 244); der wiederholte Blick in ein weiteres Exemplar der gleichen Zeitung scheint einem rational denkenden Individuum völlig ungeeignet, um den Wahrheitsgehalt zu evaluieren.

Obwohl die Praktikerliteratur zur Rhetorik schon länger darüber diskutiert, ob wiederholt dargebotene Aussagen eher geglaubt werden (z. B. Thouless 1953: 64), fand die erste empirische Überprüfung des Zusammenhangs erst Ende der 70er Jahre statt. Hasher, Goldstein und Toppino (1977) spielten dazu Probanden zu drei Zeitpunkten (mit

1 Der Toubkal ist mit 4.167m tatsächlich der höchste Gipfel im Atlasgebirge (Michael 2002: 127). 
je zwei Wochen Abstand) mehrere Aussagen auf Tonband vor, wobei die Teilnehmer den Wahrheitsgehalt jedes Statements unmittelbar nach dem Hören auf einer siebenstufigen Skala einschätzen sollten. Pro Durchgang präsentierten die Autoren 60 Aussagen, wovon 20 in jedem Folgedurchgang wiederholt und mit 40 neuen gemischt wurden. Die Rezipienten schätzten die wiederholten Statements sowohl im zweiten als auch im dritten Durchgang als glaubwürdiger ein. Der Effekt zeigte sich sowohl bei den faktisch wahren als auch bei den falschen Aussagen.

Diese erste Untersuchung zum Truth-Effekt hatte fast prototypischen Charakter: Die meisten nachfolgenden Studien wählten ein ähnliches Vorgehen ${ }^{2}$ und demonstrierten fast ausnahmslos ${ }^{3}$, dass Rezipienten dazu tendieren, eine Aussage eher zu glauben, wenn man sie wiederholt präsentiert (z. B. Arkes, Hackett \& Boehm 1989; Bacon 1979; Begg, Anas \& Farinacci 1992; Boehm 1994; Gigerenzer 1984). Auch eine aktuelle Metaanalyse bestätigt die Stabilität bisheriger Befunde (Dechêne, Stahl, Hansen \& Wänke 2010). Neben dem hier verwendeten Begriff „Truth-Effekt“ (Schwartz 1982: 394) finden sich in der Literatur noch diverse weitere Bezeichnungen für das Phänomen, wie z. B. „validity effect“ (Boehm 1994), „illusory-truth effect“ (Mitchell, Dodson \& Schacter 2005), „illusion of truth“ (Begg et al. 1992) oder „frequency-validity effect“ (Gigerenzer 1984).

\subsection{Ursachen des Truth-Effekts}

Warum führt die wiederholte Darbietung der gleichen Aussage dazu, dass man diese eher glaubt? Der Effekt basiert einerseits auf (vorwiegend) bewusst ablaufenden gedächtnisbasierten Prozessen, andererseits auf (vorwiegend) unbewusst ablaufenden vertrautheitsbasierten Prozessen. Bei den gedächtnisbasierten Prozessen ist die „convergent validity“ maßgeblich: Rezipienten glauben eine Aussage eher, wenn verschiedene Quellen diese äußern (Arkes, Boehm \& Xu 1991; Brown \& Nix 1996). Dieser Mechanismus dürfte jedoch nicht funktionieren, wenn - wie in den meisten Experimenten zum TruthEffekt - alle Aussagen von der gleichen Quelle stammen. Doch die wiederholte Präsentation eines Statements verleitet Probanden zu der irrtümlichen Annahme, die Aussage schon einmal in einem anderem Zusammenhang gehört zu haben, z. B. von Freunden oder aus den Medien (Arkes et al. 1991, 1989; Schwartz 1982). Diese Fehlattribution ist eng verbunden mit spezifischen Erklärungen für das Auftreten des Sleeper-Effekts (Hovland, Janis \& Kelley 1953; Hovland \& Weiss 1951; Kumkale \& Albarracin 2004; Weiss 1953). Gemäß der Dissociation-Hypothese wird die Verbindung von Botschaft und Quelle mit der Zeit schwächer, weshalb beide nach einer bestimmten Zeit getrennt voneinander abgerufen werden (Hovland \& Weiss 1951); die später formulierte und empirisch besser bestätigte Differential-Decay-Hypothese besagt, dass die Informationen über die Quelle einer Aussage unter bestimmten Umständen schneller vergessen werden als die Botschaft selbst (Greenwald, Pratkanis, Leippe \& Baumgardner 1986; Pratkanis, Greenwald, Leippe \& Baumgardner 1988). Es gibt verschiedene empirische

2 Diese Untersuchungen wiederholen die Statements jedoch meist nur einmal.

3 Eine der wenigen Ausnahmen bildet die Studie von Schwartz (1982), der den Truth-Effekt bei einer bestimmten Aussagengruppe nicht nachweisen kann. Dabei handelt es sich um Statements, die ursprünglich nur als Puffer zwischen den Wiederholungen der eigentlichen Stimulus-Statements („principal list“) gedacht waren, um Erinnerungseffekte abzuschwächen. Die PufferStatements („supplementary list“) werden allerdings am Ende des Experiments ebenfalls wiederholt, um eine zusätzliche Überprüfung des Truth-Effekts zu ermöglichen. Der Autor begründet nicht schlüssig, weshalb der Effekt ausbleibt. 
Evidenzen dafür, dass das Quellenvergessen bzw. die Fehlattribution der Quelle den Truth-Effekt beeinflusst (z. B. Arkes et al. 1989; Roggeveen \& Johar 2002).

Indes tritt der Truth-Effekt auch auf, wenn die Teilnehmer bei der wiederholten Beurteilung der Aussagen glauben, diese nie zuvor gehört zu haben; er zeigt sich sogar, wenn die Teilnehmer wissen, dass das Statement bereits im ersten Durchgang präsentiert wurde (Arkes et al. 1989; Bacon 1979). Eine Fehlattribution der Statements zu anderen Quellen kann diesen Mechanismus nicht erklären, weshalb man neben gedächtnisbasierten auch vertrautheitsbasierte Prozesse als Ursachen des Truth-Effekts berücksichtigt. Diese beruhen auf zwei einander folgenden Mechanismen: Erstens erhöht der wiederholte Kontakt mit einem Reiz dessen „processing fluency“, d. h. er kann in nachfolgenden Rezeptionssituationen flüssiger verarbeitet werden (Bornstein 1989; Reber, Schwarz \& Winkielman 2004; Reber, Winkielman \& Schwarz 1998). Zweitens lässt die flüssigere Verarbeitung einer Aussage ein Gefühl der Vertrautheit entstehen und das Statement deshalb glaubwürdiger erscheinen (Parks \& Toth 2006; Reber \& Schwarz 1999; Unkelbach 2007): „Statements that are easy to process are experienced as familiar [...], thus leading participants to feel that they have heard or seen this before, suggesting that it is probably true " (Reber \& Schwarz 1999: 342). Doch warum lässt die flüssigere Verarbeitung eines Statements dieses vertrauter („familiar“) wirken? Unkelbach (2006, 2007) zeigt, dass Rezipienten - wohl im Laufe ihrer Sozialisation - gelernt haben, dass die „processing fluency “ und der Wahrheitsgehalt von Botschaften korrelieren. ${ }^{4}$ So vertrauen Menschen z. B. auf die Heuristik, dass wahre Aussagen mit höherer Wahrscheinlichkeit wiederholt, hingegen Aussagen, die definitiv falsch sind, nicht weiter tradiert werden. Die beschriebenen Effekte entstehen sowohl durch die Erhöhung der perzeptuellen Geläufigkeit (z. B. empfindet man Aussagen, die aufgrund des Kontrastes von Buchstaben- und Hintergrundfarbe leichter lesbar sind, als glaubwürdiger; Reber \& Schwarz 1999; Unkelbach 2007) als auch durch die Erhöhung der konzeptuellen Geläufigkeit (z. B. leichteres Erkennen einer Aussage durch deren Einbettung in einen passenden Kontext; Parks \& Toth 2006). Entscheidend für das Auftreten des Effekts ist, dass die „processing fluency“ im Vergleich zu einem anderen Standard erhöht ist: Sind z. B. alle Statements schlecht lesbar, bewerten die Rezipienten deren Glaubwürdigkeit nicht unterschiedlich (Dechêne, Stahl, Hansen \& Wänke 2009; Hansen, Dechêne \& Wänke 2008). Erst die individuell erlebte Diskrepanz in der Verarbeitungsflüssigkeit verschiedener Aussagen lässt also den Effekt auftreten.

Sowohl die gedächtnisbasierten als auch die vertrautheitsbasierten Prozesse wirken sich auf die Einschätzung des Wahrheitsgehaltes aus. Dabei ist noch nicht geklärt, welcher der beiden Mechanismen den größeren Einfluss ausübt: Teilweise deuten die Studien darauf hin, dass die Manipulation der Quellenvielfalt („source variability“) weitaus stärkere Effekte erzielt (Arkes et al. 1989); andere Untersuchungen identifizieren vor allem einen Einfluss der „familiarity“ (Roggeveen \& Johar 2002). Das Design der jeweiligen Experimente sowie das benutzte Stimulusmaterial könnten zentrale Moderatoren dafür sein. Weiterhin ist unklar, wie sich beide Prozesse gegenseitig beeinflussen: Während z. B. Begg, Anas und Farinacci (1992) die Mechanismen völlig unabhängig konzeptualisieren, demonstrieren Arkes, Boehm und Xu (1991), dass sich diese intensiv gegenseitig beeinflussen. Weitgehend einig sind sich die Forscher, dass sowohl gedächtnisals auch vertrautheitsbasierte Prozesse unabhängig voneinander den Truth-Effekt auslösen können - in der Regel sind jedoch beide gemeinsam ursächlich.

4 Unkelbach (2007) zeigt, dass es sich bei der positiven Verknüpfung von Geläufigkeit und wahrgenommenem Wahrheitsgehalt des Stimulus um eine erlernte Assoziation handelt, die man durch Erlernen eines negativen Zusammenhangs umkehren kann. 


\subsection{Moderierende Bedingungen des Truth-Effekts: Stimulusmerkmale}

Der Truth-Effekt gilt als relativ robust und wurde bereits für verschiedene Arten von Aussagen nachgewiesen: Die meisten Studien nutzen triviales Faktenwissen bzw. relativ unbekannte Statements aus verschiedenen Wissensbereichen - wie Geografie, Geschichte, Sport, Kunst usw. - als Stimulusmaterial (Bacon 1979; Gigerenzer 1984; Hasher et al. 1977; Schwartz 1982). Die „Illusion of Truth“ zeigt sich aber auch bei Aussagen über bekannte Produkte und Marken (Hawkins \& Hoch 1992; Roggeveen \& Johar 2002, 2007) sowie bei Meinungsäußerungen (Arkes et al. 1989). Die Präsentationsform der Statements hat keinen Einfluss auf die Effektstärke: Oft legen die Forscher die Aussagen schriftlich vor (z. B. Arkes et al. 1989; Schwartz 1982), doch auch die verbale Präsentation ist relativ üblich (Bacon 1979; Gigerenzer 1984; Hasher et al. 1977). Dabei macht es keinen Unterschied, ob eine Frau oder ein Mann die Statements vorliest (Gigerenzer 1984).

Ob die Aussagen faktisch wabr oder falsch sind, spielt für das Auftreten des TruthEffekts ebenfalls keine Rolle (Gigerenzer 1984; Hasher et al. 1977). Er zeigt sich sowohl bei Statements, die anfangs als glaubwürdig beurteilt werden, als auch bei solchen, welche die Probanden zu Beginn als unglaubwürdig einstufen (Arkes et al. 1989). Die wiederholte Rezeption erhöht den angenommenen Wahrheitsgehalt von Statements sogar dann, wenn der Versuchsleiter deren Glaubwürdigkeit vorab in Frage stellt (Begg, Armour \& Kerr 1985). Hoch plausible Aussagen profitieren jedoch etwas weniger vom Truth-Effekt als Statements, die man zu Beginn als wenig glaubwürdig einstuft (Roggeveen \& Johar 2002). Völlig offensichtlich darf die Antwort auf die jeweiligen Botschaften dabei freilich nicht sein: Der Aussage, dass die Erde eine Scheibe sei, werden Versuchspersonen wohl auch nach der zehnten Präsentation nicht zustimmen. Generell ist der Truth-Effekt größer, wenn sich die Probanden bei der Beurteilung des Wahrheitsgehalts von Statements unsicher sind (Unkelbach 2007) sowie wenn die Aussagen von einer glaubwürdigen Quelle stammen (Begg et al. 1992).

Die wiederholte Darbietung der Aussagen muss noch nicht einmal im gleichen Wortlaut erfolgen. So zweifeln Probanden bei der wiederholten Präsentation z. B. auch den Wahrheitsgehalt gegensätzlich lautender Statements an (Bacon 1979). Begg, Armour und Kerr (1985) demonstrieren in diesem Zusammenhang, dass allein das Priming des entsprechenden Themengebiets einen moderaten Truth-Effekt bewirkt: Erwähnen sie beispielsweise vorab das Thema Körpertemperatur von Hennen (ohne Nennung einer spezifischen Temperatur), beurteilten die Probanden das Statement, dass die Körpertemperatur von Hennen 104 Grad Fahrenheit betrage, als glaubwürdiger. Allerdings schwächt sich der Effekt unter dieser Bedingung deutlich ab: Die Differenz zwischen neuen und wiederholten Themen bzw. Statements erreicht nur ein Fünftel der Stärke im Vergleich zur Gruppe, der man die wortgleichen Aussagen wiederholt präsentierte (Begg et al. 1985). Ein ganz ähnliches Ergebnis fanden auch Arkes, Boehm und Xu (1991: 576), die verschiedene Aussagen über China bewerten ließen: „Hearing any passage having to do with China during Week 2 caused subjects to increase their judged validity of the China sentences originally seen during Week 1 “.

Der Truth-Effekt ist relativ robust, was die Länge des Wiederholungsintervalls anbelangt. Studien lassen zwischen den Präsentationen manchmal nur wenige Minuten (Begg \& Armour 1991; Schwartz 1982), aber auch eine (Arkes et al. 1989), drei (Bacon 1979) oder fünf Wochen (Arkes et al. 1991) verstreichen. Während es keinen Unterschied macht, ob eine oder zwei Wochen zwischen den Rezeptionen liegen (Gigerenzer 1984), schwächt sich der Effekt nach drei Wochen ab und verliert nach fünf Wochen noch weiter an Stärke (Arkes et al. 1991). Wie lange die Statements präsentiert werden und wie groß 
das Zeitintervall zwischen der Präsentation der jeweiligen Statements innerhalb des Sets ist, spielt für die Effektstärke hingegen keine Rolle (Dechêne et al. 2010; Gigerenzer 1984).

Für die vorliegende Untersuchung ist insbesondere die Anzahl der Wiederbolungen relevant. Inwiefern dadurch Auftreten und Stärke des Effekts beeinflusst werden, ist nicht umfassend geklärt: Die überwiegende Mehrheit der Studien testet den Effekt, indem sie die Statements nur einmal wiederholt (z. B. Arkeset al. 1989; Bacon 1979; Begg et al. 1985; Schwartz 1982). Nur sehr wenige Analysen variieren die Präsentationshäufigkeit, so z. B. Hasher, Goldstein und Toppino (1977) in der „Ursprungsstudie“, die den Wahrheitsgehalt der Statements insgesamt dreimal beurteilen lassen. Dieser steigt zwischen der ersten und zweiten Messung deutlich an, von der zweiten zur dritten Messung gibt es nur einen moderaten Zuwachs. Auch Gigerenzer (1984) präsentiert die Statements dreimal und stellt fest, dass die Einschätzung des Wahrheitsgehalts nur zwischen der ersten und zweiten Messung signifikant zunimmt, während der Effekt zwischen der zweiten und dritten Messung ausbleibt. Arkes, Boehm und Xu (1991) testen den Einfluss der Wiederholung schließlich mit insgesamt sechs Präsentationen der gleichen Aussage, wobei zwischen den Darbietungen jeweils eine Woche liegt. Das Experiment enthüllt nur zwischen der ersten und zweiten Präsentation einen signifikanten Anstieg der Einschätzung des Wahrheitsgehalts, während die weiteren Präsentationen wirkungslos bleiben (Arkes et al. 1991). Die bislang durchgeführten Analysen deuten demnach auf Deckeneffekte hin, die bereits nach der ersten Rezeption auftreten. Indes liegen zwischen den Präsentationen der Aussagen stets mindestens sieben Tage. Die mehrstufige Manipulation der Präsentationshäufigkeit in einer sehr viel kürzeren zeitlichen Reihenfolge steht bislang aus - ob hierbei auch Deckeneffekte auftreten, der Effekt sich stärkt oder gar Reaktanzeffekte entstehen, ist also unklar.

\subsection{Moderierende Bedingungen des Truth-Effekts: Rezipientenmerkmale}

Inwiefern bestimmte soziodemographische Variablen Auftreten und Stärke des TruthEffekts beeinflussen, ist bisher kaum erforscht. Die Mehrheit der Studien zieht relativ kleine, homogene Stichproben zum Testen des Effekts heran. Meist sind die Teilnehmer allesamt Studenten, weshalb sich etwaige Unterschiede im Alter oder der Bildung ohnehin nicht prüfen lassen. Auch ob das Geschlecht die Beurteilung des Wahrheitsgehalts wiederholter Statements beeinflusst, ist nicht bekannt - doch gibt es dafür auch keine theoretischen Anhaltspunkte. Während man den Einfluss der Bildung bislang völlig außer Acht ließ, wurde zumindest der Faktor Alter schon untersucht: Law, Hawkins und Craik (1998) zeigen, dass Ältere eher annehmen, bestimmte Aussagen schon außerhalb des Experiments gehört zu haben; auch zeigt sich der Truth-Effekt bei älteren Probanden deutlicher. Andere Studien finden diesen Zusammenhang hingegen nicht (Parks \& Toth 2006), und auch die Metaanalyse von Dechêne et al. (2010) deutet nicht daraufhin, dass sich das Alter der Teilnehmer auf den Effekt auswirkt.

Den Einfluss von Elaborationstiefe und Involvement ${ }^{5}$ bei der Rezeption der Statements nahmen bereits mehrere Analysen in den Fokus (z. B. Begg et al. 1992; Begg et al. 1985; Hawkins \& Hoch 1992). Zumeist manipulierten die Forscher schlicht die Aufmerksamkeit der Probanden - und damit bestenfalls das situative Involvement. Die Probanden wurden im ersten Durchgang z. B. instruiert, entweder den Wahrheitsgehalt der präsentierten Statements zu evaluieren (high involvement) oder die Verständlichkeit der

5 Die vorliegenden Studien differenzieren nicht trennscharf zwischen Elaborationstiefe, Involvement und Aufmerksamkeit. 
Aussage zu beurteilen (low involvement). Die meisten Studien zeigen, dass der TruthEffekt unter „low-involvement-Bedingungen“ stärker ausfällt (z. B. Begg et al. 1992; Hawkins \& Hoch 1992). Hawkins und Hoch (1992; Experiment 3 ) demonstrieren jedoch auch, dass der Effekt ausbleibt, wenn man die Probanden instruiert, lediglich die Häufigkeit des Vorkommens bestimmter Buchstaben innerhalb der eingeblendeten Statements zu zählen. „Thus, there appears to be a minimum level of processing that must occur for the truth effect to take place“ (Hawkins \& Hoch 1992: 222). Wenngleich nicht alle Studien den Zusammenhang von „low involvement“ und Stärke des Truth-Effekts finden (Begget al. 1985; Experiment 4), demonstriert doch die Metaanalyse von Dechêne et al. (2010), dass der Effekt unter „low involvement“-Bedingungen in der Regel stärker wird. Darüber hinaus erkennt Bacon (1979), dass sich der Effekt abschwächt, wenn man den Probanden mitteilt, dass manche Statements im Experiment wiederholt dargeboten werden.

Eine womöglich zentrale Bedingung des Truth-Effekts ist bisher ebenfalls kaum erforscht: das Vorwissen der Rezipienten zu den jeweils erfragten Themen. Zumeist nutzen die Studien eine Bandbreite verschiedener Aussagen aus sehr unterschiedlichen Wissensbereichen (vgl. Kapitel 2.3). In solchen Analysen sind für jeden Teilnehmer sowohl Aussagen aus vertrauten als auch aus weniger bekannten Themengebieten dabei. Wie wirkt es sich aber aus, wenn Personen sich mit dem spezifischen Themengebiet auskennen bzw. über kein Vorwissen verfügen? Man könnte vermuten, dass Kenntnisse auf dem jeweiligen Gebiet dazu führen, dass die Rezipienten weniger anfällig für den TruthEffekt sind: Aufgrund ihrer Expertise lassen sie sich nicht so leicht durch einen Wiederholungseffekt täuschen. Umso mehr überrascht das Ergebnis einer Studie von Boehm (1994): Studienanfänger und fortgeschrittene Psychologiestudenten sollten den Wahrheitsgehalt von Statements aus der psychologischen Fachliteratur beurteilen: „Paradoxically, the students most knowledgable about a topic were also the most likely to exhibit the validity effect" (Boehm 1994: 290). Auch Arkes, Hackett und Boehm (1989: 90) zeigen, dass der Effekt nur bei Themen auftritt, bei denen sich die Probanden zumindest ein moderates Wissen attestierten: „The influence of repetition on rated validity was restricted to those topics about which subjects were highly or moderately knowledgeable. Topics about which subjects deemed themselves less knowledgeable did not manifest the effect". Erstaunlicherweise überprüfte man diese Befunde nicht ausführlich, und auch die Metaanalyse von Dechêne et al. (2010) setzt sich nicht damit auseinander.

\section{Zielsetzung und Hypothesen}

In der vorliegenden Untersuchung testen wir den Truth-Effekt anhand politischer Wahlkampfslogans. Der Fokus liegt einerseits auf dem themenspezifischen Vorwissen, das den Effekt moderieren könnte, andererseits auf der Rolle der Wiederholungsfrequenz. Wir vermuten, dass erst ein Mindestmaß an themenspezifischem Vorwissen den Truth-Effekt ermöglicht. Diese Annahme gründet sich auf die im vorausgehenden Kapitel präsentierten Befunde zur moderierenden Wirkung des Vorwissens der Probanden. So demonstriert Boehm (1994), dass ein geringeres themenspezifisches Wissen den Effekt abschwächt. In der Studie von Arkes, Hackett und Boehm (1989: 90) deutet sich sogar an, dass der Effekt ausbleibt, wenn man Themen präsentiert, über die die Probanden keine Kenntnisse haben.

Diesen Überlegungen liegen lernpsychologische Studien zu Grunde, wonach Wissenserwerb effizienter gelingt, wenn relevantes Vorwissen besteht und neue Informationen mit alten verknüpft werden können (Chiesi, Spilich \& Voss 1979; Johnson \& Russo 1984). Verfügt eine Person über eine gewisse Expertise in einem Bereich, steigt 
die Wahrscheinlichkeit dafür, dass sie die neuen Informationen erinnert. Bei der wiederholten Präsentation tendieren die Probanden entsprechend verstärkt zu der (irrtümlichen) Annahme, das jeweilige Statement schon einmal von einer anderen Quelle gehört zu haben (Arkes et al. 1991, 1989; Schwartz 1982). Dies schafft letztlich ein Gefühl der Vertrautheit und damit ein höheres Vertrauen in die Aussage. Wir gehen daher von einer moderierenden Rolle des themenspezifischen Vorwissens aus:

H1: Das Vorwissen der Probanden zu einem spezifischen Thema moderiert den Truth-Effekt.

H1a: Konfrontiert man Rezipienten, die (mindestens) über ein moderates Vorwissen zu dem spezifischen Thema verfügen, wiederbolt mit dem gleichen Slogan, schätzen sie dessen Wabrheitsgehalt höher ein.

H1b: Konfrontiert man Rezipienten, die über kein Vorwissen zu dem spezifischen Thema verfügen, wiederholt mit dem gleichen Slogan, wirkt sich dies nicht auf die Beurteilung des Wabrbeitsgebalts aus.

Hinsichtlich der Wirkung der Präsentationshäufigkeit kommen die wenigen Studien ${ }^{6}$, die diesen Faktor systematisch variieren, zu dem Schluss, dass mehr als eine Wiederholung entweder keinen Zuwachs der Effektstärke bewirkt (Arkes et al. 1991; Gigerenzer 1984) oder nur einen äußerst moderaten, beinahe vernachlässigbaren Effekt hervorruft (Hasher et al. 1977). Entsprechend resümieren Dechêne et al. (2010: 251) in ihrer Metaanalyse (die bei dieser Fragestellung nur drei Studien heranziehen kann, welche die Statements dreimal präsentieren), dass die wiederholte Präsentation kaum einen Einfluss auf die Einschätzung des Wahrheitsgehaltes ausübt. Obwohl die bislang ermittelten Befunde keine zusätzliche Steigerung der Glaubwürdigkeit vermuten lassen, halten wir es aus zwei Gründen für notwendig, die Präsentationshäufigkeit gegenüber früheren Studien nochmals zu überprüfen.

Erstens lassen alle bisherigen Untersuchungen, die mehr als zwei Wiederholungen durchführen, mindestens eine Woche zwischen den Präsentationen der Statements vergehen. So klafft in der Forschung zum Truth-Effekt eine Lücke, die durchaus praktische Relevanz besitzt: Wie beeinflusst es den Effekt, wenn die wiederholten Darbietungen schneller hintereinander erfolgen? Ein solches Szenario ist gerade in der Werbepraxis relevant: Man denke nur an einen mehrstündigen Fernsehabend, an dem Rezipienten die gleichen Spots innerhalb kurzer Zeit mehrfach sehen, oder an Wahlkampfzeiten, wenn Plakate mit den stets gleichen Slogans ganze Straßenzüge säumen. Hier werden Rezipienten in einem sehr kurzen Zeitraum mehrfach mit der gleichen Aussage konfrontiert. Zweitens wiederholte man - mit einer Ausnahme (Arkes et al. 1991, s. o.) - die Aussagen maximal dreimal. So stellt sich die Frage, was bei größeren Präsentationshäufigkeiten geschieht: Stärkt sich der Effekt, stellen sich Deckeneffekte ein, oder verringert sich die wahrgenommene Glaubwürdigkeit der Aussage womöglich wieder? Mit Blick auf die Präsentationshäufigkeit formulieren wir daher zusätzlich folgende Forschungsfrage:

F1: Wie schätzen Rezipienten die Glaubwürdigkeit eines Slogans ein, der sebr häufig und in kurzen zeitlichen Abständen präsentiert wird?

6 Fast alle psychologischen Untersuchungen nutzen zur Prüfung des Truth-Effekts genau eine Wiederholung der Statements (vgl. Kapitel 2.3). 


\section{Methodisches Vorgehen}

\subsection{Messung von Wiederbolungswirkungen}

Wiederholungswirkungen kann man auf vier verschiedene Arten experimentell erfassen (Dechêne et al. 2009: 1121): Erstens, indem man Effekte des gleichen Stimulus bei den gleichen Probanden wiederholt misst; die Differenz zwischen der ersten und der wiederholten Präsentation dient entsprechend als Effektstärke (within-item, within-participant). Zweitens kann man die Probanden in (mindestens) zwei Gruppen aufteilen, wobei eine Gruppe den Stimulus mehrfach betrachtet und die andere nicht; die Effektstärke bemisst sich nach der Differenz zwischen den Gruppen (within-item, betweenparticipant). Drittens kann man den gleichen Probanden einige der Stimuli wiederholt präsentieren und andere nicht; dabei beschreibt die Differenz zwischen wiederholten und neuen Stimuli den Wiederholungseffekt (between-item, within-participant). Viertens lassen sich auch die Reaktionen von Personen, die einen Stimulus wiederholt sehen, mit denen vergleichen, die einen anderen Stimulus nur einmal rezipieren (between-item, between-participant). Bei den beiden zuletzt genannten Varianten muss ein Pretest vorher sicherstellen, dass die Reaktionen auf die präsentierten Stimuli in exakt gleicher Stärke auftreten. Das zu gewährleisten, stellt strengste Anforderungen an die Auswahl der Items. Weil diese zentrale Voraussetzung der Vergleichbarkeit verschiedener Aussagen nur äußerst schwer zu erfüllen ist, sollte man besser die gleichen Aussagen präsentieren und lediglich deren Wiederholungsanzahl variieren, um sicherzustellen, dass die experimentellen Bedingungen absolut konstant sind.

Indes ist fraglich, ob man eine wiederholte Messung bei den gleichen Probanden vornehmen sollte oder besser verschiedene Gruppen vergleicht. Die Befragung der gleichen Probanden zu mehreren Zeitpunkten scheint die „Illusion of Truth“ auf den ersten Blick am besten erfassen zu können: Der Effekt postuliert ja einen Anstieg bei den gleichen Rezipienten zwischen der ersten und der wiederholten Präsentation. Entsprechend nutzen die meisten Studien zum Truth-Effekt diese Variante. Jedoch birgt sie den Nachteil, dass die wiederholte Messung die Aufmerksamkeit der Probanden sofort auf die Tatsache lenkt, dass der Wahrheitsgehalt der Aussagen erfragt wird. Die vorliegende Studie will dies vermeiden, um den Effekt unter realistischeren Bedingungen zu erfassen. Entsprechend werden wir streng nach Experimentallogik verschiedene Experimentalgruppen mit einer Kontrollgruppe vergleichen. Dieses Vorgehen wird zudem durch aktuelle Befunde zum Truth-Effekt gestützt, wonach sich der „within-item-effect“ verringert, wenn zwischen den wiederholten Präsentationen nur wenige Minuten liegen (Dechêne et al. 2010: 247). Dieser Befund überrascht keineswegs, da die Teilnehmer ihre Antworten über einen derart kurzen Zeitraum teilweise erinnern können. Aus den angeführten Gründen fiel unsere Entscheidung auf ein „within-item-between-participant"-Design.

\subsection{Design der Studie und Stimulusmaterial}

Wir konzipierten ein Online-Experiment, in dem die Teilnehmer Entwürfe verschiedener Wahlplakate hinsichtlich ihrer Farbgestaltung beurteilen sollten. Das diente als Vorwand, um den wahren Hintergrund der Studie zu verschleiern; die Hintergrundfarbe variierte zufällig, so dass von ihr keine Störeinflüsse ausgehen konnten. Die vermeintlichen Wahlplakate zeigten jeweils das Bild eines Politikers und daneben einen politischen Slogan. Die grundsätzliche Anordnung der grafischen Elemente sowie des Politikers war auf allen Bildern gleich - nur die Häufigkeit eines der Slogans variierte systematisch (s. u.). Wir wählten einen unbekannten Schweizer Politiker als Motiv und setzten ein 
Logo der Sozialdemokratischen Partei der Schweiz auf jedes der Plakate. Zudem teilten wir den (deutschen) Probanden zu Beginn der Studie mit, dass es sich um Wahlplakate aus der Schweiz handle. Die Entscheidung für den Einsatz Schweizer Wahlkampfslogans fiel aus drei Gründen: Erstens benötigten wir ein Thema, von dem ein Teil der Probanden zumindest rudimentäre Kenntnisse hat, während ein anderer Teil der Probanden nichts darüber weiß. Zweitens sollten keine umfassenden Voreinstellungen zu dem Thema bestehen: Würde man z. B. Schweizer Bürger die Slogans beurteilen lassen, so könnten die politischen Voreinstellungen der Entstehung des Effekts entgegenwirken. Drittens konnten wir durch den Verweis auf Schweizer Wahlplakate problemlos deutsche Slogans nutzen.

Die Teilnehmer betrachteten die Plakate nacheinander und beurteilten bei jedem Plakat Fragen zu dessen Farbgestaltung. Damit wollten wir sicherstellen, dass sich die Probanden mit den jeweils präsentierten Bildern zumindest kurz auseinandersetzen mussten und nicht unmittelbar weiterklickten. Unter anderem beurteilten sie die Aussage „Hintergrundfarbe, Slogan und Gesichtsausdruck des Politikers harmonieren miteinander", was die Aufmerksamkeit der Probanden zumindest partiell auch auf den Slogan lenken sollte. ${ }^{7}$ Insgesamt variierten wir parallel sechs verschiedene Statements, die eine (vermeintliche) politische Leistung der werbenden Partei wiedergaben. Fünf davon dienten lediglich der Ablenkung und wurden in jeder Gruppe jeweils nur einmal präsentiert: „Kinderbetreuung verbessert“, „Regenerative Energien gefördert“, „Staatsverschuldung gebremst“, „Integration von Migranten geglückt“, „Arbeitslosigkeit gesenkt“. Die Reihenfolge dieser Statements variierte zufällig. Als unabhängige Variable diente die Häufigkeit der Aussage „Bildungschancen verbessert“. Wir wählten dieses Item aus zwei Gründen als geeigneten Stimulus: Erstens zeigte ein Pretest, dass es verglichen mit den anderen Statements - als durchschnittlich glaubwürdig empfunden wurde. ${ }^{8}$ Zweitens wurde es sowohl von Probanden, die sich mit der Schweizer Politik rudimentär auskannten, als auch von jenen, die in dieser Hinsicht über kein Vorwissen verfügten, gleichermaßen glaubwürdig eingeschätzt. Die Häufigkeit des Auftretens des Statements variierten wir systematisch, weitgehend orientiert an bisherigen Studien zum Truth-Effekt, um in dieser Hinsicht Vergleichbarkeit herzustellen (vgl. Abschnitt 2.3). Während wir der Kontrollgruppe (KG) lediglich die fünf irrelevanten Slogans präsentierten, sah Experimentalgruppe 1 (EG1) den relevanten Slogan einmal, Experimentalgruppe 2 (EG2) dreimal und Experimentalgruppe 3 (EG3) insgesamt sechsmal. EG3 stellt mit Blick auf die bisherige Forschung eine Erweiterung dar, da sich eine sechsmalige Präsentation eines Statements bisher nur in einer Studie findet (Arkes et al. 1991) - in diesem Experiment lag jedoch stets eine Woche zwischen den Präsentationen. Dieses Intervall sollte nun deutlich kürzer gehalten werden. Der deutlichen Erhöhung der Häufigkeit liegt die Vermutung zugrunde, dass sich der Truth-Effekt nicht beliebig stei-

7 Die Anweisung, auf die Farbgestaltung der Plakate zu achten, erhöht die Wahrscheinlichkeit einer heuristischen Verarbeitung der Slogans, was wiederum das Auftreten des Truth-Effekts begünstigt (vgl. Kapitel 2.4). Dies gilt für alle experimentellen Bedingungen gleichermaßen, weshalb Unterschiede in den Versuchsgruppen nicht auf unterschiedliche Verarbeitungsmodi zurückgeführt werden können und die interne Validität gewährleistet ist. Zudem entspricht im Falle von Wahlslogans auf Plakaten eine heuristische Verarbeitung vermutlich eher der realen Rezeptionssituation, da diese in der Regel beiläufig rezipiert werden, was die externe Validität der Versuchssituation etwas vergrößert.

8 Beim Pretest schätzten 48 Studenten der Ludwig-Maximilians-Universität München die Glaubwürdigkeit der Statements ein. Die Operationalisierung erfolgte mit denselben Items, die später in der Hauptstudie verwendet wurden. 
gern lässt bzw. sich bei zu häufiger Präsentation sogar umkehren könnte. Der Versuchsaufbau bestand somit aus drei Experimentalgruppen und einer Kontrollgruppe (vgl. Abbildung 1).

\section{Abbildung 1: Versuchsaufbau}

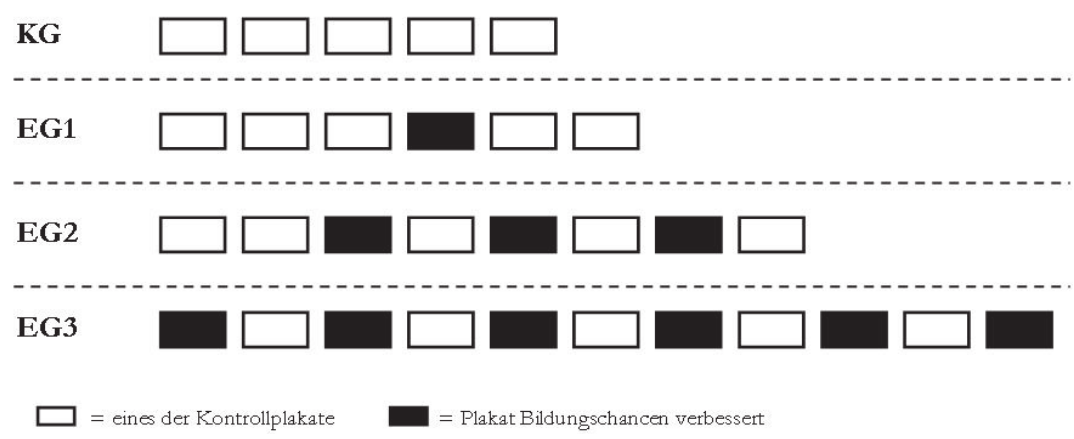

Anmerkung: Um mögliche Primacy- und Recency-Effekte zu vermeiden, präsentierten wir die Plakate innerhalb der Versuchsgruppen in zufälliger Reihenfolge.

\subsection{Messung der relevanten Konstrukte und Stichprobenzusammensetzung}

Die Messung der relevanten Konstrukte erfolgte nach dem Ende der Präsentation aller Plakate. Der Fragebogen begann mit einer längeren Batterie an Ablenkungsfragen zur Gestaltung der Plakate sowie zum dort abgebildeten Politiker. Diese Fragen waren notwendig, um einige Minuten zwischen Stimuluspräsentation und der Abfrage der relevanten Variablen verstreichen zu lassen. Als zentrale abhängige Variable diente der wahrgenommene Wahrheitsgehalt der Aussage „Bildungschancen verbessert“. Diesen erfassten wir als Mittelwert der Zustimmung zu den zwei Items „Ich halte die Aussage, dass durch die SPS die Bildungschancen verbessert wurden, für glaubwürdig“ und „Ich glaube, dass die SPS die Bildungschancen verbessert hat" (jeweils 5-stufig skaliert; Cronbachs Alpha $=.89)$. Weiterhin erfassten wir die Einschätzung des Wahrheitsgehalts zu jedem der präsentierten Kontrollslogans ebenfalls mit den jeweils gleichen Formulierungen (z. B. „Ich halte die Aussage, dass die SPS die Kinderbetreuung verbessert hat, für glaubwürdig“ bzw. „Ich glaube, dass die SPS die Kinderbetreuung verbessert hat“). Die Itembatterie präsentierten wir - wie alle übrigen Items im Fragebogen - randomisiert.

Die Kenntnisse der Schweizer Politik erfragten wir nicht über einen Test, sondern mittels der Selbsteinschätzung der Befragten. Wir nutzten keine Fragen nach dem allgemeinen Wissen über die Schweiz, weil diese auch im Sinne geographischer oder kultureller Kenntnisse gedeutet werden könnten - entsprechend würde eine Person, die häufig ihren Urlaub in der Schweiz verbringt, sich selbst große Landeskenntnisse attestieren, ohne tatsächlich über das politische Geschehen in dem Land Bescheid zu wissen. Auch die allgemeine Frage nach Kenntnissen der Schweizer Politik vermieden wir, weil sich diese im Pretest als zu unspezifisch erwies. Letztlich wählten wir die beiden Items „Ich kenne die wichtigsten Schweizer Politiker“, sowie „Ich weiß über die Parteien in der Schweiz Bescheid“. Probanden, die bei jedem der beiden Items zumindest die „2“ auf der fünfstufigen Skala angaben, galten als Personen, die sich wenigstens rudimentäre 
Kenntnisse über die Schweizer Politik attestieren. ${ }^{9}$ Weiterhin erfassten wir die Bewertung der Plakate, die Glaubwürdigkeit des abgebildeten Politikers, das politische Interesse, die Parteineigung sowie verschiedene soziodemographische Variablen.

Wir rekrutierten die Probanden über ein Online-Panel, zu dem sich Interessierte deutschlandweit freiwillig anmelden können, um an sozialwissenschaftlichen Befragungen teilzunehmen. Die im Folgenden präsentierten Ergebnisse basieren auf einer Stichprobe von insgesamt 745 Personen (KG: 204; EG1: 188; EG2: 189; EG3: 164). Die Probanden sind im Schnitt 29,3 Jahre alt (SD: 11,7), 61,4 \% sind Frauen, und beinahe $90 \%$ der Teilnehmer haben die allgemeine oder fachgebundene Hochschulreife. Der Strukturvergleich der vier Gruppen zeigt, dass sich deren Zusammensetzung hinsichtlich der soziodemographischen Merkmale sowie des politischen Interesses und der Parteineigung nicht signifikant unterscheidet. Alle Teilnehmer der Untersuchung sind deutsche Staatsbürger.

\section{Ergebnisse}

\subsection{Einfluss des themenspezifischen Vorwissens auf den Truth-Effekt}

Hypothese H1 postuliert, dass das Auftreten des Truth-Effekts vom Vorwissen der Probanden zu dem Thema moderiert wird. Vor dem Testen der Hypothese kontrollieren wir, ob die Teilnehmer, die über themenspezifisches Vorwissen verfügen, und jene, die angeben, keinerlei Kenntnisse darüber zu haben, die Glaubwürdigkeit des Slogans „Bildungschancen verbessert“ gleich beurteilen. Dazu greifen wir auf die Teilnehmer der Kontrollgruppe zurück, die den relevanten Stimulus nicht sah. Bestünde bereits hier ein Unterschied zwischen Personen mit und ohne Kenntnisse der Schweizer Politik, müsste man davon ausgehen, dass sich die Gruppen allein aufgrund ihres Vorwissens in der Einschätzung der Glaubwürdigkeit unterscheiden. Der t-Test ergibt jedoch keine signifikanten Mittelwertunterschiede zwischen beiden Gruppen $(t(194)=0.55, p>.05)$. Beide beurteilen den Wahrheitsgehalt der Aussage demnach gleich, weshalb die notwendige Voraussetzung zum Testen der ersten Hypothese erfüllt ist.

Die Prüfung der eigentlichen Hypothese erfolgt mit Hilfe einer ANOVA, in welche die Beurteilung der Glaubwürdigkeit des Slogans „Bildungschancen verbessert“ als abhängige Variable und die Zugehörigkeit zur jeweiligen Versuchsgruppe (Präsentationshäufigkeit) als echter experimenteller Faktor eingeht. Eine zweite unabhängige Variable bildet die Kenntnis der Schweizer Politik. Träfe unsere Annahme zu, dass das themenspezifische Vorwissen einen moderierenden Einfluss auf den Truth-Effekt ausübt, müsste sich dies in einem signifikanten Interaktionseffekt der beiden unabhängigen Variablen äußern. Mit zunehmender Präsentationshäufigkeit müssten Probanden mit Vorwissen höhere Glaubwürdigkeitseinschätzungen abgeben als Probanden ohne Vorwissen.

Die Analyse enthüllt sowohl einen Haupteffekt des Vorwissens zu dem Thema ( $\mathrm{F}(1$, $697)=7.70, p<.01)$ als auch einen signifikanten Interaktionseffekt von Präsentationshäufigkeit und Kenntnissen der Schweizer Politik $(\mathrm{F}(3,697)=2.69, \mathrm{p}<.05)$. Abbildung 2 zeigt, dass sich die Mittelwerte jener Probanden, die über keinerlei Vorwissen verfügen,

9 Da die Frage nach den Kenntnissen der Schweizer Politik nach der experimentellen Manipulation gestellt wurde, könnte hier prinzipiell eine Konfundierung vorliegen. Eine einfaktorielle Varianzanalyse mit der Kenntnis als abhängiger Variable zeigt allerdings, dass sich die Versuchsgruppen diesbezüglich nicht unterschieden („Ich weiß über die Parteien in der Schweiz Bescheid“, $F(3,749)=0.68, \mathrm{p}>.05$; „Ich kenne die wichtigsten Schweizer Politiker“, $\mathrm{F}(3,749)=$ $0.35, \mathrm{p}>.05)$. 
in den vier Versuchsgruppen kaum unterscheiden; hier zeigt sich demnach kein TruthEffekt. Dies bestätigt auch eine nachfolgende ANOVA, die nur mit diesen Probanden durchgeführt wurde: Sie deckt weder einen signifikanten Effekt der Gruppe $(\mathrm{F}(3,543)=$ $0.47, \mathrm{p}>$.05) noch signifikante Differenzen zwischen den einzelnen Gruppen auf. Betrachtet man hingegen die Mittelwerte jener Teilnehmer, die angaben, über ein gewisses Vorwissen zur Schweizer Politik zu verfügen, zeigt sich eine stete Zunahme der Mittelwerte von der KG über EG1 (einmalige Präsentation des Slogans) bis hin zu EG2 (dreimalige Präsentation); in EG3 (sechsmalige Präsentation) sinkt die Beurteilung der Glaubwürdigkeit der Aussage hingegen wieder ab. Eine weitere separate ANOVA auf Basis der Probanden mit Vorwissen weist einen signifikanten Effekt der Präsentationshäufigkeit $(F(3,154)=2.78, p<.05)$ auf. Signifikante Unterschiede (Bonferroni) ergeben sich hierbei zwischen KG und EG2 (MD = 0.56, $\mathrm{p}<.05)$.

Um sicherzustellen, dass der von uns gefundene Effekt kein Artefakt der „Mehrpräsentation" von Slogans ist (die Experimentalgruppen sahen jeweils insgesamt mehr Plakate als die KG, vgl. Abbildung 1), überprüfen wir zudem, ob bei den anderen Slogans, die wir jeweils nur einmal zeigten, ähnliche Effekte auftreten. Wäre dies der Fall, so wäre ein Anstieg in der Glaubwürdigkeit womöglich allein auf die höhere Anzahl der Plakate insgesamt zurückzuführen. Die dazu durchgeführte MANOVA (Glaubwürdigkeit der einmalig präsentierten Kontroll-Slogans jeweils als aV, Präsentationshäufigkeit und Vorwissen als $\mathrm{uV}$ ) zeigt weder signifikante Haupteffekte der Gruppen noch einen signifikanten Interaktionseffekt von Gruppe und themenspezifischem Vorwissen, wie er beim manipulierten Slogan zu beobachten ist. Demnach sind die berichteten Effekte auf die Manipulation der Präsentationshäufigkeit des Stimulus-Slogans und nicht auf die häufigere Präsentation von Plakaten insgesamt zurückzuführen.

Hypothese $\mathrm{H} 1$ sowie die darunter spezifizierten Hypothesen $\mathrm{H} 1 \mathrm{a}$ sowie $\mathrm{H} 1 \mathrm{~b}$ werden demnach bestätigt: Wie vermutet, wird das Auftreten des Truth-Effekts vom Vorwissen der Probanden zu dem Thema moderiert. Entsprechend Hypothese H1a führt die wiederholte Präsentation eines Slogans dazu, dass Rezipienten, die zumindest über ein moderates Vorwissen zur Schweizer Politik verfügen, diesen als glaubwürdiger empfinden. Wenngleich der Anstieg zwischen KG und EG1 relativ moderat ausfällt und nicht signifikant ist, bewirkt die dreimalige Präsentation des Statements (EG2) eine deutliche Steigerung der Glaubwürdigkeitseinschätzung. ${ }^{10}$ Hypothese H1b spezifiziert schließlich, dass die Einschätzung der Glaubwürdigkeit einer Aussage bei Rezipienten, die über kein Vorwissen zu dem Thema verfügen, durch die wiederholte Präsentation nicht beeinflusst wird. Hypothesenkonform gibt es bei jenen Teilnehmern keine signifikanten Mittelwertunterschiede. Eine mögliche Erklärung für diesen Befund liegt in lerntheoretischen und motivationalen Konzepten und wird im Fazit diskutiert.

\subsection{Einfluss der Wiederholungsanzabl auf den Trutb-Effekt}

Neben dem Einfluss von themenspezifischem Vorwissen untersuchen wir, wie sich die Glaubwürdigkeitsurteile der Probanden verändern, wenn eine Aussage sehr häufig in kurzer zeitlicher Abfolge präsentiert wird. Dazu führen wir - als Erweiterung bisheriger Studien - eine vierte Faktorstufe ein, bei der die Versuchsteilnehmer den Stimulus-Slogan sechsmal sehen (EG3). Wie in Abbildung 2 dargestellt, bleibt die Manipulation bei Personen ohne Vorkenntnisse der Schweizer Politik erneut ohne Wirkung, wohingegen die Glaubwürdigkeitseinschätzungen der Probanden mit Vorkenntnissen absinken, und

10 Dass diese Beurteilung im Vergleich zu EG3, der wir das Statement insgesamt sechsmal präsentierten, wieder absinkt, diskutieren wir unter Hypothese H2. 
Abbildung 2: Beurteilung der Glaubwürdigkeit der Aussage „Bildungschancen verbessert" in den vier Versuchsgruppen

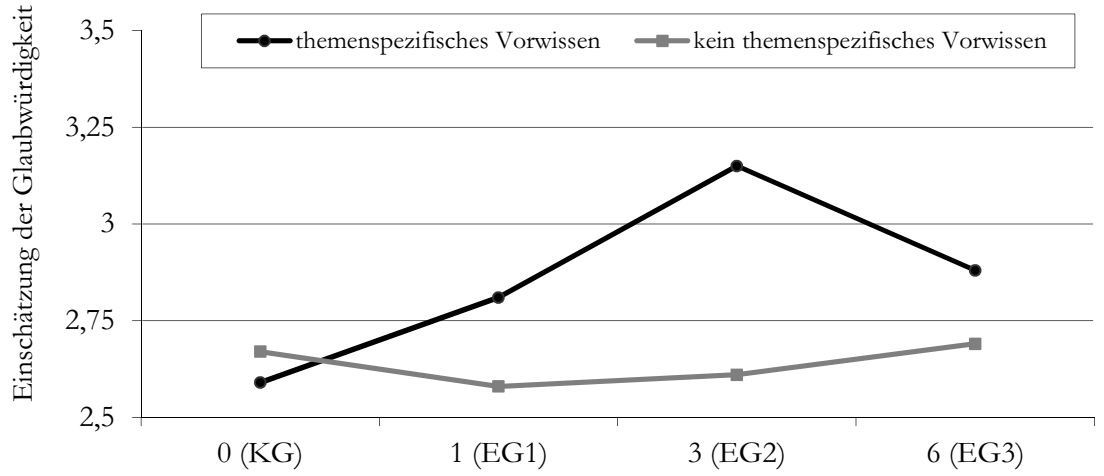

Präsentationshäufigkeit des Stimulus-Slogans

zwar so weit, dass sich kein signifikanter Unterschied mehr zur Kontrollgruppe ergibt $(\mathrm{MD}=0.29, \mathrm{p}>.05)$. Das Ergebnis deckt sich mit den Befunden früherer Studien, die zeigen, dass es sich beim Truth-Effekt nicht um eine beliebig zu steigernde Größe handelt, sondern bereits nach wenigen Wiederholungen eine „Sättigungsgrenze“ erreicht wird und Deckeneffekte auftreten (Arkes et al. 1991; Gigerenzer 1984).

Darüber hinaus deuten die vorliegenden Ergebnisse sogar in die umgekehrte Richtung, d. h. bei sechsmaliger Präsentation wird der Wahrheitsgehalt des Stimulus-Slogans wieder geringer eingeschätzt. Da sich allerdings die Mittelwerte zwischen EG2 und EG3 ebenfalls nicht signifikant unterscheiden, können wir nicht sagen, ob es sich um einen Deckeneffekt (aufgrund eines wear-outs) oder um ein tatsächliches Absinken der Glaubwürdigkeit handelt. Letzteres wäre, sowohl bezogen auf unsere Datenlage als auch auf die bisherige Forschung zu Wiederholungswirkungen, ein plausibles Szenario: Der oben angeführte Vergleich der Mittelwerte von EG2 und EG3 sowie zwischen KG und den beiden EGs lässt weder einen linearen Zusammenhang von Präsentationshäufigkeit und Glaubwürdigkeit der Aussage noch einen Deckeneffekt wahrscheinlich erscheinen - das deutliche, wenngleich nicht signifikante Absinken der Glaubwürdigkeit deutet eher auf Reaktanz hin (Brehm 1966). Diese könnte entstehen, weil ein Teil der Probanden die mehrmalige Präsentation in sehr kurzer Zeit bemerkt und den Persuasionsversuch durchschaut (Friestad \& Wright 1994). Die so entstehende umgekehrte U-Kurve, die sich in Abbildung 2 andeutet, ist bei Wiederholungswirkungen ein häufig gefundenes Bild: So verbessern sich beispielsweise die Einstellungen gegenüber einem präsentierten Stimulus zunächst mit steigender Wiederholungsanzahl und schwächen sich ab einem bestimmten Wiederholungslevel ${ }^{11}$ wieder ab (Batra \& Ray 1986; Berlyne 1970; Cacioppo \& Petty 1979). Zukünftige Studien sollten diesen Zusammenhang weiter analysieren.

11 Die Anzahl hängt von verschiedenen Faktoren, wie der Komplexität des Stimulus sowie der Aufmerksamkeit der Rezipienten, ab. 


\section{Fazit}

Die permanente Wiederholung gleicher Aussagen ist ein hervorstechendes Charakteristikum der Mediengesellschaft, das sich am deutlichsten in der Werbung zeigt, allerdings keineswegs nur auf diese beschränkt ist (Hawkins \& Hoch 1992: 223). Auch politische Akteure wiederholen in Pressemitteilungen, politischen Diskussionsrunden, redaktionellen Texten oder Interviews bestimmte Aussagen immer wieder. Ziel des Beitrags war es herauszufinden, wie solche Wiederholungen auf die Glaubwürdigkeit der jeweiligen Aussagen wirken. Aufbauend auf der psychologischen Forschung zum Truth-Effekt variierten wir in einem Experiment die Präsentationshäufigkeit eines politischen Slogans systematisch und erfassten die Effekte auf die wahrgenommene Glaubwürdigkeit der Statements.

Entsprechend unserer Annahme stellte sich der Truth-Effekt auch bei politischen Slogans ein, allerdings nur bei Personen, die bereits über ein mit dem Aussageninhalt verbundenes themenspezifisches Vorwissen verfügen. Die Ursache dafür kann einerseits an der durch das Vorwissen erleichterten Informationsverarbeitung liegen, wodurch neue Aussagen besser erinnert und abgerufen werden können (Chiesi et al. 1979: 270). Je mehr inhaltsbezogenes Vorwissen besteht, desto eher werden neue Informationen mit alten verknüpft und entsprechend effizienter und elaborierter gespeichert. Andererseits geht ein erhöhtes Vorwissen auch mit einem größeren Interesse an der Thematik einher; das Interesse steuert Aufmerksamkeit und Intention, mit der die Versuchspersonen die Wahlplakate betrachteten (Petty \& Cacioppo 1986). Dies könnte erklären, warum der Truth-Effekt in der vorliegenden Studie nicht bei Personen auftritt, die über kein themenspezifisches Vorwissen verfügen; auch diese sollten ja - im Laufe des Experiments - mit dem Stimulus-Slogan „vertraut“ werden. Wir erklären das Ausbleiben des Effekts bei jenen Teilnehmern über die spezifische Aufgabenstellung: Sie hatten die Aufgabe, die Plakate hinsichtlich Farbgestaltung und Design zu beurteilen. Man kann mutmaßen, dass sich Personen ohne jedes Vorwissen völlig auf diese Aufgabenstellung beschränkten und den Slogans zu wenig Aufmerksamkeit widmeten. Personen mit themenspezifischem Vorwissen könnten die Aussagen hingegen stärker fokussiert haben. So deuten unsere Befunde, ebenso wie die von Hawkins \& Hoch (1992), darauf hin, dass ein Mindestmaß an Aufmerksamkeit eine notwendige Bedingung für das Auftreten des TruthEffekts ist.

Arkes, Hackett und Boehm (1989: 90) veranschaulichen die oben genannten Mechanismen an einem konkreten Beispiel: Zwar würden auch Kunstfans den Wahrheitsgehalt der Aussage „The females in Salvador Dali's paintings were usually modeled by his wife" kaum einschätzen können, doch könnte es ihnen - im Vergleich zu Personen, die sich gar nicht mit Kunst auskennen - leichter fallen, sich das Statement zu merken. Zudem haben Kunstfans ein größeres Interesse an der präsentierten Aussage; daher sind sie motivierter, diese zu verarbeiten und sich daran zu erinnern. Das Beispiel demonstriert, dass Interesse und Vorwissen eng miteinander verwoben sind: Wer über Vorwissen zu einem Thema verfügt, ist meist an weiteren Informationen darüber interessiert; wer großes Interesse an einem Thema hat, wird sich andererseits leichter und vermehrt Wissen dazu aneignen. Sowohl das themenspezifische Interesse als auch das Vorwissen steuern demnach die Aufmerksamkeit sowie Speicherungs- und Abrufprozesse. Wenngleich Interesse und Vorwissen in der Realität zumeist stark korrelieren, könnten zukünftige Studien beide separat manipulieren.

Hinsichtlich der Wirkung von sehr häufigen Wiederholungen der gleichen Botschaft bleiben unsere Befunde uneindeutig. Zwar weisen die Ergebnisse auf einen Glaubwürdigkeitsrückgang hin, wenn die gleiche Aussage sehr oft präsentiert wird - allerdings 
sind statistisch keine gesicherten Schlüsse möglich, ob es sich um einen tatsächlichen Rückgang oder nur um einen Deckeneffekt handelt. Während die Forschung bislang von schnell eintretenden Deckeneffekten oder einem linearen Zusammenhang von Präsentationshäufigkeit und Glaubwürdigkeit der Aussage ausgeht, deuten unsere Befunde eher auf eine umgekehrte U-Kurve hin.

Im Gegensatz zu sämtlichen Vorgängerstudien legten wir den Probanden die relevanten Aussagen nicht isoliert vor, sondern in einem realistischeren Kontext in Form eines Wahlplakates. Dennoch ist die externe Validität des Experiments freilich eingeschränkt. Erstens weicht die Stichprobe in verschiedenen Merkmalen (z. B. Bildung, Alter) erheblich vom Bevölkerungsdurchschnitt ab und ist keineswegs repräsentativ. Zweitens handelt es sich beim Stimulusmaterial zwar um professionell gestaltete Wahlplakate, im Gegensatz dazu war die Rezeptionssituation aber vergleichsweise unrealistisch, da die „Plakate“ den Probanden auf einem Computerbildschirm und integriert in einen Online-Fragebogen präsentiert wurden. Folglich sind die Befunde nicht mit der Wirkung echter Wahlplakate gleichzusetzen. Wenn überhaupt, ähnelte die Art der Präsentation Werbebannern auf Internetseiten. Die Einbettung der Aussage in den Kontext politischer Werbung führt allerdings auch dazu, dass der Truth-Effekt wahrscheinlich schwächer ausfällt als bei einem anderen Kommunikator. So schätzen Rezipienten die Aussagen von Politikern einerseits und der Werbung andererseits als wenig glaubwürdig ein und der Truth-Effekt schwächt sich daher ab (Begg et al. 1992). Umso mehr erstaunt es, dass die Glaubwürdigkeit der Slogans trotzdem von der Wiederholung profitierte.

Aus den vorliegenden Befunden und Grenzen unserer Untersuchungsanlage ergeben sich mit Blick auf zukünftige Studien einige interessante Fragestellungen, die vor allem die Aussagenquelle sowie die Wiederholungsanzahl und, damit verbunden, das Persuasionswissen der Rezipienten betreffen. Nachfolgende Untersuchungen könnten insbesondere Anzahl und Verschiedenartigkeit der Aussagequellen manipulieren und Auswirkungen auf die Glaubwürdigkeit der kommunizierten Aussagen erfassen. Auch aus diesem Blickwinkel wird die gesellschaftliche Relevanz des Truth-Effekts deutlich, z. B. bei der Diskussion um publizistische Vielfalt, d. h. sowohl die Vielfalt der Quellen als auch der Inhalte und ihre Rolle für die Meinungsbildung in der Bevölkerung. Führt beispielsweise Konsonanz dazu, dass Menschen bestimmte Sachverhalte und Standpunkte für wahr halten und sie daher zur Grundlage ihres Handelns machen? Die zweite Frage betrifft die Wiederholungsanzahl: Unsere Vermutung einer umgekehrten U-Kurve scheint vor dem Hintergrund bisheriger Befunde zu Wiederholungswirkungen durchaus plausibel. Indes sind dringend Anschlussstudien notwendig, die erstens überprüfen, ob der Kurvenverlauf tatsächlich derart gestaltet ist. Zweitens sollten Folgeuntersuchungen das Persuasionswissen der Rezipienten berücksichtigen.

\section{Literatur}

Arkes, H. R., Boehm, L. \& Xu, G. (1991): Determinants of Judged Validity. Journal of Experimental Social Psychology, 27(6), 576-605.

Arkes, H. R., Hackett, C. \& Boehm, L. (1989): The generality of the relation between familiarity and judged validity. Journal of Behavioral Decision Making, 2(2), 81-94.

Ayer, A. J. (1986). Can there Be a Private Language? In S. Shanker (Hrsg.), Ludwig Wittgenstein: critical assessments (S. 239-247). Dover, NH: Croom Helm.

Bacon, F. T. (1979): Credibility of Repeated Statements: Memory for Trivia. Journal of Experimental Psychology: Human Learning and Memory, 5(3), 241-252.

Batra, R. \& Ray, M. L. (1986): Situational Effects of Advertising Repetition: The Moderating Influence of Motivation, Ability, and Opportunity to Respond. Journal of Consumer Research, 12(4), 432-445. 
Begg, I. \& Armour, V. (1991): Repetition and the Ring of Truth: Biasing Comments. Canadian Journal of Behavioural Science, 23(2), 195-213.

Begg, I., Anas, A. \& Farinacci, S. (1992): Dissociation of Processes in Belief: Source Recollection, Statement Familiarity, and the Illusion of Truth. Journal of Experimental Psychology: General, 121(4), 446-458.

Begg, I., Armour, V. \& Kerr, T. (1985): On Believing What We Remember. Canadian Journal of Behavioural Science, 17(3), 199-214.

Berlyne, D. E. (1970): Novelty, Complexity, and Hedonic Value. Perception E Psychophysics, 8(5), 279-286.

Boehm, L. E. (1994): The Validity Effect: A Search for Mediating Variables. Personality and Social Psychology Bulletin, 20(3), 285-293.

Bornstein, R. F. (1989): Exposure and Affect: Overview and Meta-Analysis of Research, 1968-1987. Psychological Bulletin, 106(2), 265-289.

Brehm, J. W. (1966): A Theory of Psychological Reactance. New York: Academic Press.

Brown, A. S. \& Nix, L. A. (1996): Turning Lies into Truths: Referential Validation of Falsehoods. Journal of Experimental Psychology: Learning, Memory, and Cognition, 22(5), 1088-1100.

Cacioppo, J. T. \& Petty, R. E. (1979): Effects of Message Repetition and Position on Cognitive Responses, Recall, and Persuasion. Journal of Personality and Social Psychology, 37(1), 97-109.

Chiesi, H. L., Spilich, G. J. \& Voss, J. F. (1979): Acquisition of Domain-Related Information in Relation to High and Low Domain Knowledge. Journal of Verbal Learning and Verbal Behavior, 18(3), 257-273.

Dechêne, A., Stahl, C., Hansen, J. \& Wänke, M. (2009): Mix Me a List: Context Moderates the Truth Effect and the Mere-Exposure Effect. Journal of Experimental Social Psychology, 45(5), 1117-1122.

Dechêne, A., Stahl, C., Hansen, J. \& Wänke, M. (2010): The Truth About the Truth: A MetaAnalytic Review of the Truth Effect. Personality and Social Psychology Review, 14(2), 238-257.

Friestad, M. \& Wright, P. (1994): The Persuasion Knowledge Model: How People Cope with Persuasion Attempts. Journal of Consumer Research, 21(1), 1-31.

Gigerenzer, G. (1984): External Validity of Laboratory Experiments: The Frequency-Validity Relationship. American Journal of Psychology, 97(2), 185-195.

Greenwald, A. G., Pratkanis, A. R., Leippe, M. R. \& Baumgardner, M. H. (1986): Under What Conditions Does Theory Obstruct Research Progress? Psychological Review, 93(2), 216-229.

Hansen, J., Dechêne, A. \& Wänke, M. (2008): Discrepant Fluency Increases Subjective Truth. Journal of Experimental Social Psychology, 44(3), 687-691.

Hasher, L., Goldstein, D. \& Toppino, T. (1977): Frequency and the Conference of Referential Validity. Journal of Verbal Learning and Verbal Behavior, 16(1), 107-112.

Hawkins, S. A. \& Hoch, S. J. (1992): Low-Involvement Learning: Memory Without Evaluation. Journal of Consumer Research, 19(2), 212-225.

Hovland, C. I. \& Weiss, W. (1951): The Influence of Source Credibility on Communication Effectiveness. Public Opinion Quartertly, 15(4), 635-650.

Hovland, C. I., Janis, I. L. \& Kelley, H. H. (1953): Communication and Persuasion. Psychological Studies of Opinion Change. New Haven, CT: Yale University Press.

Johnson, E. J. \& Russo, J. E. (1984): Product Familiarity and Learning New Information. Journal of Consumer Research, 11(1), 542-550.

Kumkale, G. T. \& Albarracin, D. (2004): The Sleeper Effect in Persuasion: A Meta-Analytic Review. Psychological Bulletin, 130(1), 143-172.

Law, S., Hawkins, S. A. \& Craik, F. I. M. (1998): Repetition-Induced Belief in the Elderly: Rehabilitating Age-Related Memory Deficits. Journal of Consumer Research, 25(2), 91-107.

Michael, T. (2002): Diercke Weltatlas. Braunschweig: Westermann.

Mitchell, J. P., Dodson, C. S. \& Schacter, D. L. (2005): fMRI Evidence for the Role of Recollection in Suppressing Misattribution Errors: The Illusory Truth Effect. Journal of Cognitive Neuroscience, $17(5), 800-810$.

Orwell, G. (1987): 1984. Boston, MA: Houghton Mifflin Harcourt.

Parks, C. M. \& Toth, J. P. (2006): Fluency, Familiarity, Aging, and the Illusion of Truth. Aging, Neuropsychology, and Cognition, 13(2), 225-253. 
Petty, R. E. \& Cacioppo, J. T. (1986): The Elaboration Likelihood Model of Persuasion. In L. Berkowitz (Hrsg.), Advances in experimental social psychology (S. 123-205). New York: Academic Press.

Pratkanis, A. R., Greenwald, A. G., Leippe, M. R. \& Baumgardner, M. H. (1988): In Search of Reliable Effects: III. The Sleeper Effect is Dead. Long Live the Sleeper Effect. Journal of Personality and Social Psychology, 54(2), 203-218.

Reber, R. \& Schwarz, N. (1999): Effects of Perceptual Fluency on Judgments of Truth. Consciousness and Cognition, 8(3), 338-342.

Reber, R., Schwarz, N. \& Winkielman, P. (2004): Processing Fluency and Aesthetic Pleasure: Is Beauty in the Perceiver's Processing Experience. Personality and Social Psychology Review, 8(4), 364-382.

Reber, R., Winkielman, P. \& Schwarz, N. (1998): Effects of Perceptual Fluency on Affective Judgments. Psychological Science, 9(1), 45-48.

Roggeveen, A. L. \& Johar, G. V. (2002): Perceived Source Variability versus Familiarity: Testing Competing Explanations for the Truth Effect. Journal of Consumer Psychology, 12(2), 81-91.

Roggeveen, A. L. \& Johar, G. V. (2007): Changing False Beliefs from Repeated Advertising: The Role of Claim-Refutation Alignment. Journal of Consumer Psychology, 17(2), 118-127.

Schwartz, M. (1982): Repetition and Rated Truth Value of Statements. American Journal of Psychology, 95(3), 393-407.

Thomas, W. I. \& Thomas, D. S. (1928): The Child in America: Behavior Problems \& Programs. New York, NY: Alfred A. Knopf.

Thouless, R. H. (1953): Straight and Crooked Thinking. London: Pan Books.

Unkelbach, C. (2006): The Learned Interpretation of Cognitive Fluency. Psychological Science, 17(4), 339-345.

Unkelbach, C. (2007): Reversing the Truth Effect: Learning the Interpretation of Processing Fluency in Judgments of Truth. Journal of Experimental Psychology: Learning, Memory, and Cognition, 33(1), 219-230.

Weiss, W. (1953): A Sleeper Effect in Opinion Change. Journal of Abnormal and Social Psychology, 48(2), 173-180. 